Solid State Communications, Vol. 44, No. 5, pp. 653--656, 1982.

Printed in Great Britain.

\title{
HYBRID CYCLOTRON-INTERSUBBAND RESONANCE IN THIN InAs LAYERS CONFINED BETWEEN GaSb
}

\author{
J.C. Maan and Ch. Uihlein \\ Max-Planck-Institut für Festkörperforschung, Hochfeld-Magnetlabor, 166X, F-38042 Grenoble-Cédex, France \\ and \\ L.L. Chang and L. Esaki \\ IBM Thomas J. Watson Research Center, P.O. Box 218, Yorktown Heights, NY 10598, U.S.A. \\ (Received 4 May 1982 by A. R. Miedema)
}

\begin{abstract}
In a heterostructure of InAs confined between GaSb, measurements of far infrared transmission as a function of the magnetic field and its orientation demonstrate the formation of hybrid subbands due to electric and magnetic quantization. The observed energies of resonant transitions with the magnetic field parallel to the layer plane are in quantitative agreement with those calculated theoretically.
\end{abstract}

IN TWO-DIMENSIONAL electron systems, like those at semiconductor surfaces and interfaces or on liquid helium, the electrons are mobile in a plane and confined in the perpendicular direction, which leads to the formation of two-dimensional subbands. A magnetic field $(B)$ perpendicular to the plane splits each electron subband into Landau levels, and transitions between these levels, such as Cyclotron Resonance (CR), can be observed. As $B$ is tilted away from the perpendicular direction, the presence of a field component parallel to the plane mixes the electrostatic and magnetic quantization and hybrid subbands are formed. In this paper we report clear evidence for this hybrid subband formation from magnetotransmission experiments in a heterostructure of thin $(1000 \AA)$ InAs layers confined between $\mathrm{GaSb}$. The evolution of the spectra with the angle between the magnetic field and the plane of the layer show that not only the perpendicular field component determines the resonance position. Specifically, for $B$ parallel to the layer plane sharp transmission minima, corresponding to the hybrid subband transitions are observed with energies deviating from the bulk as predicted theoretically.

The Hamiltonian of an electron confined in the $y z$ plane by a potential $V(x)$ in a magnetic field $B \| z$ is given by:

$\frac{\hbar^{2}}{2 m^{*}}\left[-\frac{\partial^{2}}{\partial x^{\prime 2}}+\frac{x^{\prime 2}}{l^{4}}+\frac{2 m^{*}}{\hbar^{2}} V(x)\right] \psi(x)=E \psi(x)$,

$x^{\prime}=x+\frac{\hbar k_{y}}{e B}$,

with $m^{*}$ the effective mass, $l=\sqrt{ } \mathbf{h} / e B$ the cyclotron radius and $E$ the energy eigenvalue. The influence of a parallel magnetic field on the energy level structure depends on the relative magnitude of the last two terms in equation (1), for a given $l$ and characteristic length $x_{0}$ of a certain potential. If the last term dominates the magnetic field it can be treated as a perturbation as in $\mathrm{Si}$ inversion layers [1,2] or electrons on liquid helium [3], where a broadening and a shift of the intersubband resonance, with additional side peaks at $\pm h \omega_{c}$ where $\omega_{c}$ is the cyclotron frequency $[4,5]$ is observed in a parallel magnetic field. CR itself is shown, however, to depend only on the perpendicular component of $B$ [6]. For $\mathrm{PbTe}$ inversion layers the magnetic field term dominates [7] and a three-dimensional behaviour of CR is observed. If the two terms are comparable hybrid subbands are formed whose energies depend on the magnetic field and this has led to the observation of quantum oscillations in the magnetoresistance in an In As accumulation layer in the parallel field configuration [8]. In this case the effect of the potential $V(x)$ in equation (1) is to lift the $k_{y}$ degeneracy or, otherwise stated, the energy depends on the position of the center coordinate of the CR orbit $\hbar k_{y} / e B$.

The sample used in the present experiments was grown by molecular beam epitaxy, consisting of alternating layers of $1000 \AA$ InAs and $1000 \AA \mathrm{GaSb}$. The far infrared (FIR) magnetotransmission experiments were performed at $5 \mathrm{~K}$ by using a number of fixed radiation energies while varying $B$ up to $20 \mathrm{~T}$ with orientations between parallel and perpendicular to the plane of the layers.

At the interface the $\mathrm{GaSb}$ valence band is $150 \mathrm{meV}$ higher in energy than the InAs conduction band, resulting in electron transfer from $\mathrm{GaSb}$ to InAs. The dipole field across the interface due to the transferred charge bends the bands. Potential wells are created by the relative position of the bands together with their bending, giving rise to two-dimensional accumulation layers at the interface with electron subbands in InAs and hole subbands in $\mathrm{GaSb}$. 

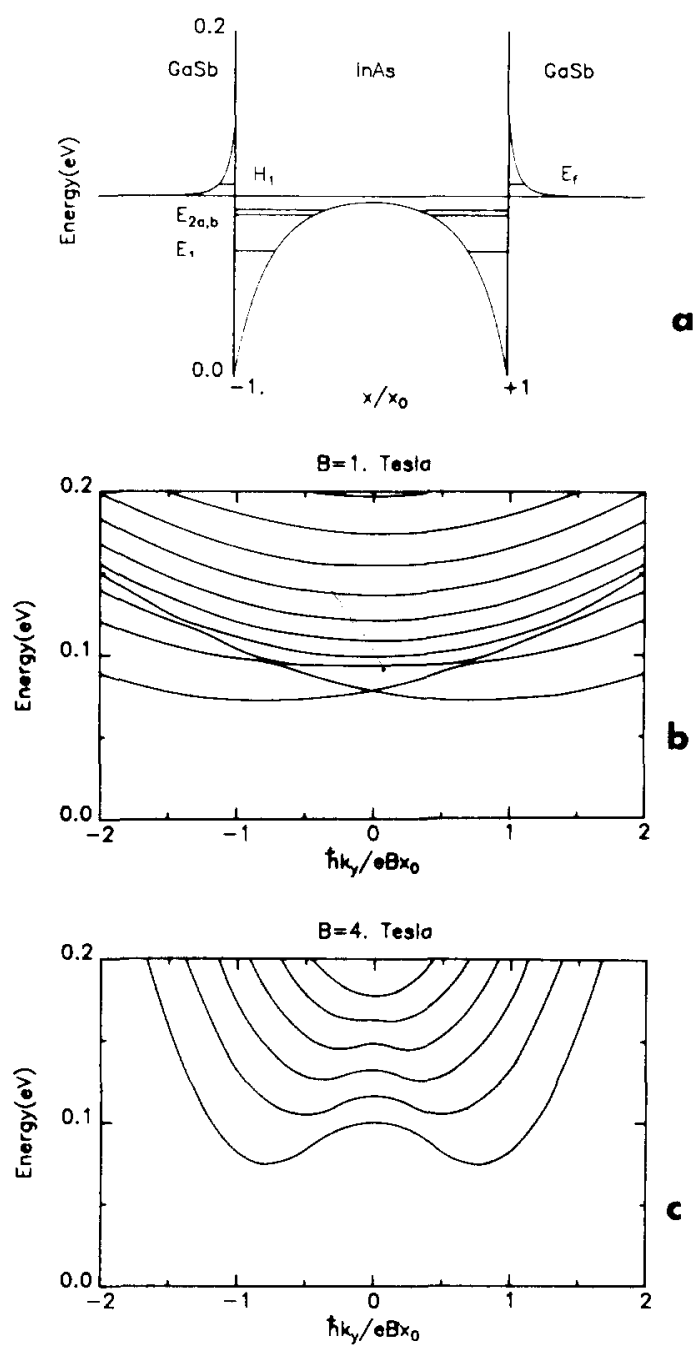

Fig. 1. (a) Potential profile of a $1000 \AA$ InAs layer confined between $\mathrm{GaSb}$, with the calculated subbands $E_{1}$, $E_{2 a, b}$ and $H_{1}$, indicated, $x_{0}$ is characteristic length of the potential well. The Fermi energy is at $104 \mathrm{meV}$. (b) and (c) The dispersion of the electron subbands in a parallel magnetic field of 1 and $4 \mathrm{~T}$ respectively; $\hbar k_{y} / e B$ is the cyclotron resonance orbit center coordinate, and is taken to be zero in the middle of the layer.

In a simplified Thomas-Fermi approximation the potential profile of a heterojunction is [9] given by,

$V(x)=E_{F}\left[1-1 /\left(1+\frac{x}{x_{0}}\right)^{4}\right]$,

where $x_{0}$ is the characteristic length which depends on the Fermi energy $E_{F}$ and materials parameters. $V(x)$ of the present heterostructure is obtained by superimposing the potential of the two interfaces and shown in Fig.

1(a). Equation (1) is solved exactly for zero magnetic field for a certain $E_{F}$, and the electron and hole densities are calculated. This procedure is repeated until a value of $E_{F}$ is found which satisfies the condition of charge

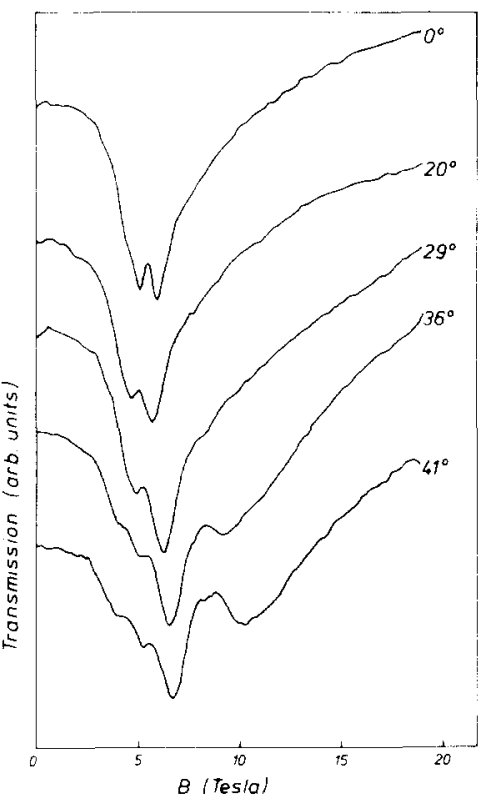

Fig. 2. Measured transmission at $57 \mu \mathrm{m}(21.7 \mathrm{meV})$ radiation of the magnetic field for different angles between the field and the normal of the layers.

neutrality. One heavy hole subband $H_{1}=108 \mathrm{meV}$, and essentially two electron subbands $E_{1}=72 \mathrm{meV}$ and $E_{2 a, b}=93,96 \mathrm{meV}$ with $E_{F}=104 \mathrm{meV}$ are obtained in this fashion. For $\mathrm{GaSb} x_{0}$ is $\sim 100 \AA$ which is much less than the layer thickness while for InAs $x_{0}$ is $\sim 500 \AA$ about half of the layer thickness. Therefore the system can be seen as that of InAs layers confined between $\mathrm{GaSb}$. This energy level structure has been confirmed by Shubnikov-de Haas and magnetoabsorption measurements in the perpendicular configuration $[10,11]$. A comparison of the last two terms in equation (1) shows that for a parallel field of the order of $5 \mathrm{~T}$, the first (magnetic) term has a negligible effect on the $\mathrm{GaSb}$ subband but that both terms are important for InAs because its mass is 14 times lighter than and the well is five times wider than that of $\mathrm{GaSb}$.

Figure 2 shows the results of the angular dependence of the FIR transmission at $57 \mu \mathrm{m}(21.7 \mathrm{meV})$. For $B \| x$, the oscillations are attributed primarily to interband transitions between Landau levels of $H_{1}$ and $E_{1}$ with the pronounced doublet to $\mathrm{CR}$ of the different two electron subbands [11]. It is known that different subbands give rise to different $\mathrm{CR}$ in materials with band non-parabolicity [12]. As the sample is rotated from $B \| x$ to $41^{\circ}$ off the $x$-axis the CR doublet becomes less defined; one part shifting slightly to higher fields while the other becoming somewhat weakened and hardly moving with the angle. Both these and the $H_{1}-E_{1}$ transitions are broadened in general. At about $20^{\circ}$ a new transition appears at $\sim 10 \mathrm{~T}$ as can be seen in Fig. 2 , 

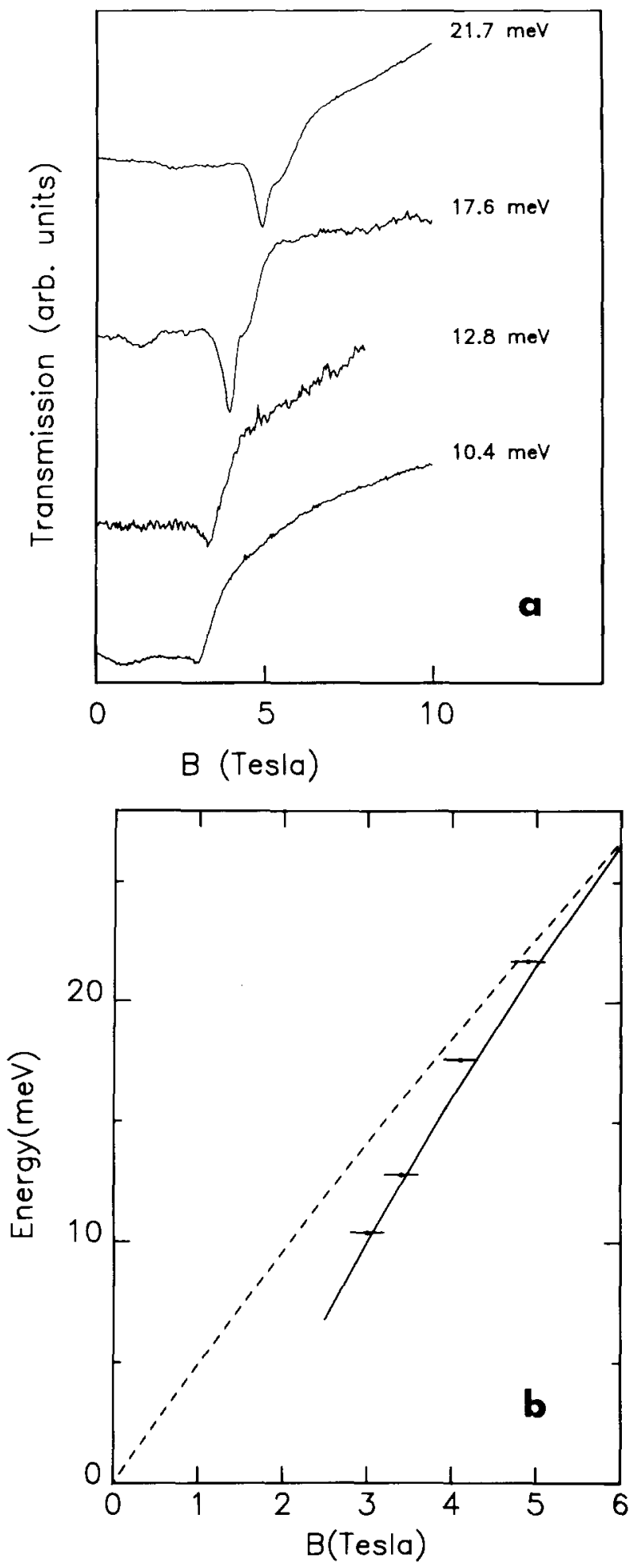

Fig. 3. (a) Measured transmission for different radiation energies as a function of the magnetic field parallel to the layers. (b) Plot of the position of the sharp transition in $a$. The dotted line is the calculated CR position in bulk InAs and the solid line is the calculated hybrid subband transition with the cyclotron orbit center in the middle of the InAs layer. which is not observed at lower radiation energies. In Fig. 3(a) the transmission data for $B \| z$ are shown. A single sharp resonance is seen with some weak structure both at the high and the low field side. At lower radiation energies $(<7 \mathrm{meV})$, the transmission becomes increasing monotonically with the field. A plot of the position of the sharp resonance vs. radiation energy is shown in Fig. 3(b). The transition energies do not extrapolate to the origin of the energy scale and, with increasing fields, tend to approach the calculated CR energies of bulk InAs.

The results for the $B \| z$ configuration are analyzed by solving equation (1) exactly for the potential profile in the InAs layer shown in Fig. 1. As was said before the effect of $V(x)$ in equation (1) is to cause the energy to depend on the position of the CR orbit center. Figures 1(b) and (c) show the level structure for 1 and $4 \mathrm{~T}$ as a function of $C=\hbar k_{y} / e B x_{0}$ the CR center coordinate normalized to the characteristic length of the well $x_{0}$. At $4 \mathrm{~T}$ and $C=0$ a regular energy spacing is observed which is close to $\hbar \omega_{c}$, the Landau level spacing a while at $C=1$ the level splitting is about $2 \hbar \omega_{c}$. These values of $\hbar \omega_{c}$ and $2 \hbar \omega_{c}$ are eventually reached for higher magnetic fields. At 1 T Fig. 1 shows a rather complex level structure.

Physically the results can be understood in the following way. At high fields the magnetic potential dominates the electrostatic one. This leads to Landau like levels at the center and magnetic surface states at the interface. Such magnetic surface states have an energy separation of $2 \hbar \omega_{c}$ [13] for the CR orbit center at the surface. At low fields electric and magnetic subbands are completely mixed. The magnetic field lifts the degeneracy of the lowest $E_{1}$ subband of the two wells, because it breaks the symmetry for positive and negative motion along the interface. The $E_{2 a}$ and $E_{2 b}$ subbands, which are already split by the interaction of the two wells, evolve into hybrid subbands with increasing fields. Since different levels have a different dispersion they show an anticrossing behaviour as can clearly be seen in Fig. 1(b).

Assuming a selection rule $\Delta k_{y}=0$ sharp transitions can be expected between parallel levels adjacent to the Fermi energy. Therefore the position of the sharp transitions in Fig. 3(a) is compared with the calculated energy difference at $C=0$ in Fig. 3(b). The agreement between calculated and measured data is quite satisfying and indicates clearly that transitions of hybrid subbands, and not simple CR, are observed. The reason that no sharp resonance is observed below $2 \mathrm{~T}$ can now be understood. As can be seen from Fig. 1(b) at low magnetic fields there are no parallel bands across the Fermi energy and transitions at these magnetic fields must be very broad.

It should be noted in this connection that the 
observed transition is in fact sharper than would intuitively be expected since, as is clear from Fig. 1(b) the bands in the vicinity of $C$ are not completely equally spaced. However, the observed linewidth will be the result of all possible transitions weighted with their transition probabilities, which need not to be equal for different $C$ values. The weak structure at fields below the sharp transition, in Fig. 3 (a) may well be due to transitions at $C \neq 0$. These transitions, as expected should be broad, have a stronger magnetic field dependence, and occur at a lower field. The broad shoulder at the high field side cannot be explained on this basis, and we can only speculate that it may arise from a combined hybrid intersubband spin transition.

Qualitatively the results of the angular dependence can now be understood. As the tilt angle increases the effect of the $B_{z}$ component becomes more important. For instance it is clear that $H_{1}-E_{1}$ broadens and is not at all observed $B \| z$. The effect of $B_{z}$ is negligible on $H_{1}$ but significant on $E_{1}$ which has a strong dispersion, resulting in the broadening and eventual disappearance of the transition. In addition the doublet due to CR of different subbands weakens and disappears as $B_{z}$ increases, since the distinction between different electric subbands loses its meaning. For $B \| z$ only one single hybrid inter-subband transition exists. The new transition observed at large angles at $10 \mathrm{~T}$ in Fig. 2 probably has its origin associated with an $E_{1}-E_{2 a, b}$ transition. Their energies differ by $21-24 \mathrm{meV}$ and vary different with $B_{z}$ to account for the angular dependence.

In summary it can be concluded that the measurements reported here clearly show the formation of hybrid electric-magnetic subbands in a two-dimensional heterostructure under the influence of a parallel magnetic field. The results are analyzed in detail and a quantitative agreement is obtained between measured and calculated values.

Acknowledgements - We would like to thank C.A. Chang for growing the crystals and W. Schmid for technical assistance. The work at the IBM Thomas J. Watson Research Center was partially supported under an Army Research Office contract.

\section{REFERENCES}

1. F. Stern \& W.E. Howard, Phys. Rev. 163, 816 (1967).

2. T. Ando, J. Phys. Soc. Japan 39, 411 (1975).

3. C.L. Zipfel, T.R. Brown \& C.C. Grimes, Surf. Sci. 58, 283 (1976).

4. W. Beinvogl, A. Kamgar \& J.F. Koch, Phys. Rev. B14, 1610 (1976).

5. W. Beinvogl \& J.F. Koch, Phys. Rev. Lett. 40, 1736 (1978).

6. G. Abstreiter, J.P. Kotthaus \& J.F. Koch, Phys. Rev. B14, 2480 (1978).

7. H. Schaber \& R.E. Doezema, Phys. Rev. B20, 5257 (1979).

8. R.E. Doezema, M. Nealon \& S. Whitmore, Phys. Rev. Lett. 45, 1593 (1980).

9. R.W. Keyes, Comments Solid State Phys. 7, 53 (1976).

10. L.L. Chang, N.J. Kawai, E.E. Mendez, C.A. Chang \& L. Esaki, Appl. Phys. Lett. 38, 30 (1980).

11. Y. Guldner, J.P. Vieren, P. Voisin, M. Voos, J.C. Maan, L.L. Chang \& L. Esaki, Solid State Commun. 41, 755 (1982).

12. A. Daerr, J.P. Kotthaus \& J.F. Koch, Solid State Commun. 17, 455 (1975).

13. M. Wanner, R.E. Doezema \& U. Strom, Phys. Rev B12, 2883 (1975). 